\title{
New Application for Generalized Regularized Long Wave (GRLW) Equation, Modified Dispersive Water Wave (MDWW) Equation and Kawahara Equation by Homogeneous Balance Method
}

\author{
${ }^{1}$ Wafaa M. Taha, ${ }^{2}$ Israa A. Ibrahim and ${ }^{3}$ M.S.M. Noorani \\ ${ }^{1}$ Department of Math., College for Sciences, Kirkuk University, Kirkuk, Iraq \\ ${ }^{2}$ Department of Math., College Education for Pure Sciences, Tikrit University, Tikrit, Iraq \\ ${ }^{3}$ School of Math. Sciences, Universiti Kebangsaan Malaysia (UKM), Selangor, Malaysia
}

\begin{abstract}
This research study examines the homogeneous balance method to obtain an exact solution of travelling wave non-linear equations. The proposed homogeneous balance method is used to obtain new solutions for Generalized Regularized Long Wave (GRLW) equation, Modified Dispersive Water Wave (MDWW) equation and Kawahara equation. Many solitary wave solutions are calculated from the solution by the hyperbolic function when parameters were taken as special values. The obtained results are compared with the F-expansion method solution and $\left(\mathrm{G}^{\prime} / \mathrm{G}\right)$-expansion method solution. The comparison reveals that our obtained results are identical to the F-expansion method and $\left(\mathrm{G}^{\prime} / \mathrm{G}\right)$-expansion method solutions when certain hypothesis is adopted. Maple Software is used to plot the 3D graph of the obtained exact solution.
\end{abstract}

Key words: Homogeneous balance method, generalized regularized long wave equation, modified dispersive water wave equation, Kawahara equation and solitary wave solutions, obtained results, hypothesis

\section{INTRODUCTION}

There is a wide range of nonlinear phenomena accord in nature and obtaining a solution for such phenomena have a significant impact, especially in physics and applied mathematics. When studying nonlinear physical phenomena, the exact solution of nonlinear evolution equation has a major influence on this types of study (Zayed and Arnous, 2012). There are several algebraic methods to calculate exact travelling wave solution was proposed in the literature. The most important and appropriate method called homogeneous balance method. This method was first proposed by Wang (1995), Wang et al. (1996) then further developed by many researchers (Elwakil et al., 2004; Fan, 2000, 2003; Khalfallah, 2009; Zhao et al., 2006). According to Fan (2000) applied homogeneous balance method was used to calculate solution for Becklund transformation when an assumption was made in the reduction of the nonlinear partial differential equation. Fan proves that there is a close relationship between the HB method, Clarkson Kruskal (CK) method and Weiss Tabor Carnevale (WTC) method. A new algebraic method was proposed by Fan (2003). The suggested method was used to calculate a new solution for solitary wave of NLPDEs and many other nonlinear evolution equations. The obtained solution has a significant impact in many areas such as applied mathematics and theoretical physics (Antonova and Biswas, 2009; Biswas, 2010; Razborova et al., 2013). The obtained solution can be expressed as a polynomial in an element that satisfies general Riccati equation and Elwakil et al. (2003). When the homogeneous balance method was applied an auto-Backlund transformation for the generalized shallow water wave equation and generalized variable coefficient $2 \mathrm{D} \mathrm{KdV}$ equation, new exact solitary wave solutions was calculated. This idea of this research study depends on the characteristic of travelling wave solution of nonlinear equations where this solution can be expressed by a polynomial in $\mathrm{u}=\Sigma^{\mathrm{N}}{ }_{\mathrm{i}=0} \mathrm{q}_{\mathrm{i}} \psi^{\mathrm{i}}, \mathrm{u}=\mathrm{u}(\xi)$ such that $\xi=\mathrm{kx}+\mathrm{wt}$. The degree of this polynomial can be calculated from considering the homogenous balance between the highest order derivatives and the nonlinear term appearing in a giving equation. The coefficient of the consider polynomial can be resulted from solving a set of algebraic equation appeared during the process of using the proposed method (Wang et al., 2008). The significance of the homogeneous balance method is making the calculation of shock or solitary type of solution easy. A general exact solution of the nonlinear equations of the Generalized Long Wave (GRLW), the (MDWW) equation and Kawahera equation was obtained. 


\section{MATERIALS AND METHODS}

Mechanism of homogeneous balance method: Consider we have a nonlinear evolution equation in the form of Eq. 1:

$$
\mathrm{F}\left(\mathrm{u}, \mathrm{u}_{\mathrm{t}}, \mathrm{u}_{\mathrm{z}}, \mathrm{u}_{\mathrm{xt}}, \mathrm{u}_{\mathrm{zx}}, \ldots,\right)=0
$$

where, $\mathrm{F}$ is a polynomial in $\mathrm{u}=\mathrm{u}(\mathrm{x}, \mathrm{t})$ and its PDEs in which the highest order derivatives and nonlinear terms are involved. The main steps of applying the homogenous balance method can be illustrated in the following points (Zayed and Arnous, 2012; Zayed and Alurrfi, 2014):

Step 1: Using the wave transformation Eq. 2:

$$
\mathrm{u}(\mathrm{x}, \mathrm{t})=\mathrm{u}(\xi), \xi=\mathrm{kx}+\mathrm{wt}
$$

Reduce Eq. 1 to the following ODE in Eq. 3:

$$
\mathrm{P}\left(\mathrm{u}, \mathrm{u}^{\prime}, \mathrm{u}^{\prime \prime}, \ldots,\right)=0
$$

where, $\mathrm{P}$ is a polynomial in $\mathrm{u}(\xi)$ and its general derivatives while $\mathrm{k}$ and $\mathrm{w}$ are constants.

Step 2: We suppose that Eq. 3 has a solution shown in Eq. 4:

$$
u(\xi)=\sum_{i=0}^{N} q_{i} \psi^{i}
$$

where, $q_{i}(i=0,1, \ldots, N)$ are constants to be calculated, such that $\mathrm{q}_{\mathrm{N}} \neq 0$ and $\psi(\xi)$ is the solution of Eq. 5 :

$$
\psi^{\prime}(\xi)=\psi^{2}(\xi)-\psi(\xi)
$$

Equation 5 has the solution:

$$
\psi(\xi)=\frac{1}{1 \pm \mathrm{e}^{\xi}}
$$

Step 3: We calculate all the derivatives of $u^{\prime}, u^{\prime \prime}, \ldots$, of the polynomial $u(\xi)$ which results:

$$
\begin{aligned}
& \mathrm{u}=\mathrm{q}_{0}+\mathrm{q}_{1} \psi+\mathrm{q}_{2} \psi^{2}+ \\
& \mathrm{u}^{\prime}=(\psi-1) \psi\left[\mathrm{q}_{1}+2 \mathrm{q}_{2} \psi+\ldots\right] \\
& \mathrm{u}^{\prime \prime}=(\psi-1) \psi\left[(-1-2 \psi) \mathrm{q}_{1}+2 \psi(-2+3 \psi) \mathrm{q}_{2}+\ldots\right] \\
& \mathrm{u}^{\prime \prime \prime}=(\psi-1) \psi\left[\left(1-6 \psi+6 \psi^{2}\right) \mathrm{q}_{1}+2 \psi(4-15 \psi+\right. \\
& \left.\left.12 \psi^{2}\right) \mathrm{q}_{2}+\ldots\right] \\
& \mathrm{u}^{(4)}=(\psi-1) \psi\left[\left(-1+14 \psi-36 \psi^{2}+24 \psi^{3}\right) \mathrm{q}_{1}+\right. \\
& \left.2 \psi\left(-8+57 \psi-108 \psi^{2}+60 \psi^{3}\right) \mathrm{q}_{2}+\ldots\right] \\
& \mathrm{u}^{(5)}=(\psi-1) \psi\left[\left(-1-30 \psi+150 \psi^{2}-240 \psi^{3}+\right.\right. \\
& \left.120 \psi \psi^{4}\right) \mathrm{q}_{1}+2 \psi\left(16-165 \psi+660 \psi^{2}-\right. \\
& \left.\left.840 \psi^{3}+360 \psi \psi^{4}\right) \mathrm{q}_{2}+\ldots\right]
\end{aligned}
$$

Step 4: We determine the positive integer $\mathrm{N}$ in Eq. 4 by considering the homogeneous balance between the highest order derivatives and the nonlinear terms in Eq. 3.

Step 5: Substitute Eq. 4 into Eq. 3, we calculate all the necessary derivatives $u^{\prime}, u^{\prime}, \ldots$, of the equation $u(\xi)$. As a result of this substitution, we obtain a polynomial of $(\psi)^{\mathrm{i}}$ $(i=0,1,2, \ldots, N)$. In this polynomial, we gather all terms of same powers and equating them to zero, we obtain a system of algebraic equations which can be solved by the Maple to get the unknown parameters $q_{i}(i=$ $0,1, \ldots, N), k$ and $\mathrm{w}$ consequently, we get the exact solutions of Eq. 1.

\section{Application of homogeneous balance method Generalized Regularized Long Wave (GRLW) equation:} In this study, our proposed method is applied to obtain a new and more general exact solution of the Generalized Regularized Long Wave (GRLW) equation read (Abazari, 2010):

$$
\mathrm{u}_{\mathrm{t}}+\mathrm{u}_{\mathrm{x}}+\mathrm{a}\left(\mathrm{u}^{2}\right)_{\mathrm{x}}-\mathrm{bu}_{\mathrm{xxt}}=0
$$

where, $\mathrm{a}$ and $\mathrm{b}$ are positive constants. Equation 7 was first proposed as a model for small amplitude long waves on the surface of the water in a channel by Peregrine (1996) and then by Benjamin et al. (1972). In physics phenomena such as unidirectional waves propagation in a water channel, long-crested waves in near shore zones and many other, the Generalized Regularized Long Wave (GRLW) equation works as an alternative model to the $\mathrm{KdV}$ equations. Suppose the following Eq. 8:

$$
\mathrm{u}(\mathrm{x}, \mathrm{t})=\mathrm{u}(\xi), \xi=\mathrm{kx}+\mathrm{wt}
$$

we obtain:

$$
(\mathrm{w}+\mathrm{k}) \mathrm{u}^{\prime}+\mathrm{ak}\left(\mathrm{u}^{2}\right)^{\prime}-\mathrm{bk}^{2} \mathrm{wu}^{\prime \prime \prime}=0
$$

Integrating Eq. 9 once with respect to $\xi$ and the setting the integration constant as zero yields:

$$
(\mathrm{w}+\mathrm{k}) \mathrm{u}+\mathrm{aku}^{2}-\mathrm{bk}^{2} \mathrm{w}^{2} \mathrm{u}^{\prime \prime}=0
$$

Now, we are balancing between $\mathrm{u}^{2}$ and $\mathrm{u}$ " then we obtain $\mathrm{N}+2=2 \mathrm{~N}$, then, $\mathrm{N}=2$, suppose that Eq. 10 has the following formal solution:

$$
\mathrm{u}(\xi)=\mathrm{q}_{0}+\mathrm{q}_{1} \psi+\mathrm{q}_{2} \psi^{2}
$$


Table 1: Comparison of proposed solution with solution of ( $\left.\mathrm{G}^{3} / \mathrm{G}\right)$-expansion method (Abazari, 2010)

The solution of $\left(\mathrm{G}^{\prime} / \mathrm{G}\right)$-expansion method

If $\lambda=1, c_{1}=1, c_{2}=0$ then, Eq. (34a) becomes

If $\mathrm{b}>0, \mathrm{a}>0$ then, Eq. 12 becomes

$u(x, t)=\frac{-3 b^{2}}{2 a\left(b k^{2}-1\right)} \operatorname{sech}^{2}\left(\frac{k x}{2}+\frac{k}{2\left(b k^{2}-1\right)} t\right)$

$u(x, t)=\frac{-3 b^{2}}{2 a\left(b k^{2}-1\right)} \operatorname{sech}^{2}\left(\frac{k x}{2}+\frac{k}{2\left(b k^{2}-1\right)} t\right)$

Substituting Eq. 11 and the derivatives in step 3 and collecting all terms with the same order of $\left(\mathrm{e}^{\xi}\right)$, we have the following:

$$
\begin{aligned}
& (\psi)^{0}: \mathrm{akq}_{0}^{2}+\mathrm{kq}_{0}+\mathrm{wq}_{0}=0 \\
& (\psi)^{1}:-\mathrm{bk}^{2} \mathrm{wq}_{1}+2 \mathrm{akq}_{0} \mathrm{q}_{2}+2 \mathrm{kq}_{1}+\mathrm{q}_{1} \mathrm{w}=0 \\
& (\psi)^{2}: 3 \mathrm{bk}^{2} \mathrm{q}_{1} \mathrm{w}-4 \mathrm{bk}^{2} \mathrm{q}_{2} \mathrm{w}+2 \mathrm{akq}_{0} \mathrm{q}_{2}+\mathrm{akq}_{1}^{2}+\mathrm{kq}_{2}+ \\
& \mathrm{q}_{2} \omega=0 \\
& (\psi)^{3}:-2 \mathrm{bk}^{2} \mathrm{q}_{1} \mathrm{w}+10 \mathrm{bk}^{2} \mathrm{q}_{2} \mathrm{w}+2 \mathrm{akq}_{1} \mathrm{q}_{2}=0 \\
& (\psi)^{4}:-6 \mathrm{bk}^{2} \mathrm{q}_{2} \mathrm{w}+\mathrm{akq}_{2}^{2} \mathrm{w}=0
\end{aligned}
$$

And solving the system of equation by using to maple, we obtain Table 1.

\section{Status 1:}

$$
\begin{aligned}
& q_{0}=0, q_{1}=\frac{-6 \mathrm{bk}^{2}}{a\left(\mathrm{bk}^{2}-1\right)} q_{2}=\frac{6 \mathrm{bk}^{2}}{\mathrm{a}\left(\mathrm{bk}^{2}-1\right)}, \mathrm{w}=\frac{\mathrm{k}}{\mathrm{bk}^{2}-1} \\
& \mathrm{u}(\mathrm{x}, \mathrm{t})=\frac{-3 \mathrm{bk}^{2}}{2 \mathrm{a}\left(\mathrm{bk}^{2}-1\right)} \operatorname{sech}^{2}\left(\frac{\mathrm{kx}}{2}+\frac{\mathrm{k}}{2\left(\mathrm{bk}^{2}-1\right)} \mathrm{t}\right)
\end{aligned}
$$

\section{Status 2:}

$$
\begin{aligned}
& \mathrm{q}_{0}=\frac{-\mathrm{bk}^{2}}{\mathrm{a}\left(\mathrm{bk}^{2}+1\right)}, \mathrm{q}_{1}=\frac{6 \mathrm{bk}^{2}}{\mathrm{a}\left(\mathrm{bk}^{2}+1\right)}, \mathrm{q}_{2}=\frac{-6 \mathrm{bk}^{2}}{\mathrm{a}\left(\mathrm{bk}^{2}+1\right)}, \\
& \mathrm{w}=\frac{-\mathrm{k}}{\mathrm{bk}^{2}+1} \\
& \mathrm{u}(\mathrm{x}, \mathrm{t})=\frac{-\mathrm{bk}^{2}}{\mathrm{a}\left(\mathrm{bk}^{2}+1\right)}+\frac{3 \mathrm{bk}^{2}}{2 \mathrm{a}\left(\mathrm{bk}^{2}+1\right)} \operatorname{sech}^{2}\left(\frac{\mathrm{kx}+\frac{-\mathrm{k}}{\mathrm{bk}^{2}+1}}{2} \mathrm{t}\right)
\end{aligned}
$$

The MDWW equation: The MDWW equations will be studied in this study (Alzaidy, 2013; Neyrame et al., 2010) by the form of the following Eq. 13 and 14 :

$$
\begin{gathered}
\mathrm{u}_{\mathrm{t}}=-\frac{1}{4} \mathrm{v}_{\mathrm{xx}}+\frac{1}{2}(\mathrm{uv})_{\mathrm{x}} \\
\mathrm{v}_{\mathrm{t}}=-\mathrm{u}_{\mathrm{xx}}-2 \mathrm{uu}_{\mathrm{zx}}+\frac{3}{2} \mathrm{vv}_{\mathrm{x}}
\end{gathered}
$$

The traveling wave variables as:

$$
\mathrm{u}(\mathrm{x}, \mathrm{t})=\mathrm{u}(\xi), \mathrm{v}(\mathrm{x}, \mathrm{t})=\mathrm{v}(\xi), \xi=\mathrm{x}-\mathrm{ct}
$$

Converting Eq. 16 and 17 into ODEs for $\mathrm{u}(\mathrm{x}, \mathrm{t})=\mathrm{u}(\xi)$ and $\mathrm{v}(\mathrm{x}, \mathrm{t})=\mathrm{v}(\xi)$ as follow:

$$
\begin{aligned}
& \frac{1}{4} \mathrm{v}^{\prime \prime}-\frac{1}{2}(\mathrm{uv})^{\prime}-\mathrm{cu} \mathrm{u}^{\prime}=0 \\
& \mathrm{u}^{\prime \prime}+2 \mathrm{uu} \mathrm{u}^{\prime}-\frac{3}{2} \mathrm{vv}^{\prime}-\mathrm{cv}^{\prime}=0
\end{aligned}
$$

where, $\mathrm{k}$ and $\mathrm{w}$ are the wave number and the wave speed, respectively. On integrating Eq. 17 and 18 with respect to $\xi$ once, yields:

$$
\frac{1}{4} \mathrm{v}^{\prime}-\frac{1}{2}(\mathrm{uv})-\mathrm{cu}=0
$$

$$
\mathrm{u}^{\prime}+\mathrm{u}^{2}-\frac{3}{2} \mathrm{~V}^{2}-\mathrm{cv}=0
$$

where, $\mathrm{c}$ is constant, suppose that the solution of the ODEs Eq. 19 and 20 can be expressed by polynomials in the terms of as follows:

$$
\begin{aligned}
& u(\xi)=\sum_{i=0}^{N} a_{i}(\psi)^{i} \\
& v(\xi)=\sum_{i=0}^{M} b_{i}(\psi)^{i}
\end{aligned}
$$

where, $a_{i}(i=0,1, \ldots, N)$ and $b_{i}(i=0,1, \ldots, M)$ are arbitrary constant and we balancing between the highest order derivatives and the nonlinear term in Eq. 21 and 22, we get $\mathrm{N}=\mathrm{M}=1$, thus, we have:

$$
\begin{aligned}
& u(\xi)=a_{0}+a_{1} \psi, a_{1} \neq 0 \\
& v(\xi)=b_{0}+b_{1} \psi, b_{1} \neq 0
\end{aligned}
$$

where, $\mathrm{a}_{0}, \mathrm{a}_{1}, \mathrm{~b}_{0}$ and $\mathrm{b}_{1}$ are constant to be determine later. substituting Eq. 23 and 24 with derivatives in step 3 and collecting all terms with the same power of $\psi$ and setting them to zero, we have the following system of algebraic equation: 


$$
\begin{aligned}
& (\psi)^{0}: \mathrm{ca}_{0}+\frac{1}{2} \mathrm{a}_{0} \mathrm{~b}_{0}=0 \\
& (\psi)^{1}: \mathrm{ca}_{1}+\frac{1}{4} \mathrm{~b}_{1}+\frac{1}{2} \mathrm{a}_{0} \mathrm{~b}_{1}+\frac{1}{2} \mathrm{a}_{1} \mathrm{~b}_{0}=0 \\
& (\psi)^{2}:-\frac{1}{4} \mathrm{~b}_{1}+\frac{1}{2} \mathrm{a}_{1} \mathrm{~b}_{1}=0 \\
& (\psi)^{3}: \frac{3}{4} \mathrm{~b}_{0}^{2}-\mathrm{a}_{0}^{2}+\mathrm{cb}_{0}=0 \\
& (\psi)^{4} \mathrm{a}_{1}-\mathrm{blc}-2 \mathrm{a}_{0} \mathrm{a}_{1}+\frac{3}{2} \mathrm{~b}_{0} \mathrm{~b}_{1}: \\
& (\psi)^{5}:-\mathrm{a}_{0}^{2}+\frac{3}{4} \mathrm{~b}_{1}^{2}-\mathrm{a}_{1}=0
\end{aligned}
$$

Solving the above algebraic equations from $(\psi)^{0}$ into $(\psi)^{5}$ by using the maple, yields.

\section{Status 1:}

$\mathrm{a}_{0}=0, \mathrm{a}_{1}=1 / 2, \mathrm{~b}_{0}=0, \mathrm{~b}_{1}=1, \mathrm{c}=-1 / 2$

$$
\begin{aligned}
& \mathrm{u}(\mathrm{x}, \mathrm{t})=\frac{1}{4}-\frac{1}{4} \tanh \left(\frac{\mathrm{x}-\frac{1}{2} \mathrm{t}}{2}\right) \\
& \mathrm{v}(\mathrm{x}, \mathrm{t})=\frac{1}{2}-\frac{1}{2} \tanh \left(\frac{\mathrm{x}-\frac{1}{2} \mathrm{t}}{2}\right)
\end{aligned}
$$

\section{Status 2:}

$\mathrm{a}_{0}=0, \mathrm{a}_{1}=1 / 2, \mathrm{~b}_{0}=0, \mathrm{~b}_{1}=-1, \mathrm{c}=1 / 2$

$$
\begin{aligned}
& \mathrm{u}(\mathrm{x}, \mathrm{t})=\frac{1}{4}+\frac{1}{4} \tanh \left(\frac{\mathrm{x}+\frac{1}{2} \mathrm{t}}{2}\right) \\
& \mathrm{v}(\mathrm{x}, \mathrm{t})=\frac{1}{2}-\frac{1}{2} \tanh \left(\frac{\mathrm{x}+\frac{1}{2} \mathrm{t}}{2}\right)
\end{aligned}
$$

\section{Status 3:}

$\mathrm{a}_{0}=1 / 2, \mathrm{a}_{1}=-1, \mathrm{~b}_{0}=1, \mathrm{~b}_{1}=0, \mathrm{c}=-1 / 2$

$$
\mathrm{u}(\mathrm{x}, \mathrm{t})=\frac{1}{2} \tanh \left(\frac{\mathrm{x}+\frac{1}{2} \mathrm{t}}{2}\right)
$$

\section{Status 4:}

$a_{0}=1 / 2, a_{1}=-1, b_{0}=-1, b_{1}=0, c=-1 / 2$

$$
\mathrm{u}(\mathrm{x}, \mathrm{t})=\frac{1}{2} \tanh \left(\frac{\mathrm{x}-\frac{1}{2} \mathrm{t}}{2}\right)
$$

\section{Status 5:}

$a_{0}=1 / 2, a_{1}=-1, b_{0}=-1, b_{1}=0, c=-1 / 2$

$$
\begin{aligned}
& \mathrm{u}(\mathrm{x}, \mathrm{t})=-\frac{1}{4}-\frac{1}{4} \tanh \left(\frac{\mathrm{x}+\frac{1}{2} \mathrm{t}}{2}\right) \\
& \mathrm{v}(\mathrm{x}, \mathrm{t})=\frac{1}{2}+\frac{1}{2} \tanh \left(\frac{\mathrm{x}+\frac{1}{2} \mathrm{t}}{2}\right)
\end{aligned}
$$

Status 6:

$a_{0}=-1 / 2, a_{1}=1 / 2, b_{0}=-1, b_{1}=1, c=1 / 2$

$$
\mathrm{u}(\mathrm{x}, \mathrm{t})=-\frac{1}{4}-\frac{1}{4} \tanh \left(\frac{\mathrm{x}+\frac{1}{2} \mathrm{t}}{2}\right)
$$

$$
\mathrm{v}(\mathrm{x}, \mathrm{t})=-\frac{1}{2}-\frac{1}{2} \tanh \left(\frac{\mathrm{x}+\frac{1}{2} \mathrm{t}}{2}\right)
$$

Kawahara equation: Let us consider the so-called Kawahara equation (Ozis and Aslan, 2010; Wazwaz, 2006):

$$
\mathrm{u}_{\mathrm{t}}+\alpha u \mathrm{u}_{\mathrm{x}}+\beta \mathrm{u}_{\mathrm{xxx}}-\gamma \mathrm{u}_{\mathrm{xxxxx}}=0
$$

where, a-c is not equal zero arbitrary constants. Equation 36, proposed first by Kawahara (1972) and this equation is a model equation for plasma waves, capillary-gravity water waves (Bridges and Derks, 2002). Moreover, this equation characterize the water waves with surface tension (Benjamin et al., 1972) (Table 2).

Let us now solve Eq. 35 by applying the homogeneous balance method. To this end, we apply the wave transformation Eq. 2 to reduce Eq. 36 to the following $\mathrm{ODE}$ :

$$
\mathrm{wu}^{\prime}+\alpha \mathrm{k}^{2} \mathrm{uu} \mathrm{u}^{\prime}+\beta \mathrm{k}^{3} \mathrm{u}^{\prime \prime \prime}-\gamma \mathrm{k}^{5} \mathrm{u}^{(5)}=0
$$

And integrating the resulting ordinary differential equation once, result:

$$
w u+\alpha k^{2}\left(\frac{u^{2}}{2}\right)+\beta k^{3} u^{\prime \prime}-\gamma k^{5} u^{(4)}=0
$$

Balancing $\mathrm{u}^{2}$ with $\mathrm{u}^{(4)}$ yields $\mathrm{N}=4$. Consequently, Eq. 37 has the formal solution:

$$
\mathrm{u}=\mathrm{q}_{0}+\mathrm{q}_{1} \psi+\mathrm{q}_{2} \psi^{2}+\mathrm{q}_{3} \psi^{3}+\mathrm{q}_{4} \psi^{4}
$$


Table 2: Comparison of proposed solution with solution of $\left(\mathrm{G}^{3} / \mathrm{G}\right)$-expansion method (Neyrame et al., 2010)

The solution of $\left(\mathrm{G}^{\prime} / \mathrm{G}\right)$-expansion method

Proposed homogeneous balance solution

If $\mathrm{c}_{1}=1, \mathrm{c}_{2}=0, \lambda=1, \mathrm{c}=1 / 2$ then Eq. 12 becomes

If $\mathrm{c}>0$ then, Eq. 33 and Eq. 34 becomes

$\mathrm{u}(\mathrm{x}, \mathrm{t})=-\frac{1}{4}-\frac{1}{4} \tanh \left(\frac{\mathrm{x}-\frac{1}{2} \mathrm{t}}{2}\right)$

$\mathrm{u}(\mathrm{x}, \mathrm{t})=-\frac{1}{4}-\frac{1}{4} \tanh \left(\frac{\mathrm{x}-\frac{1}{2} \mathrm{t}}{2}\right)$

$\mathrm{v}(\mathrm{x}, \mathrm{t})=-\frac{1}{2}-\frac{1}{2} \tanh \left(\frac{\mathrm{x}-\frac{1}{2} \mathrm{t}}{2}\right)$

$\mathrm{v}(\mathrm{x}, \mathrm{t})=-\frac{1}{2}-\frac{1}{2} \tanh \left(\frac{\mathrm{x}-\frac{1}{2} \mathrm{t}}{2}\right)$

where, $\mathrm{q}_{0}-\mathrm{q}_{4}$ are constants such that $\mathrm{q}_{4} \neq 0$, now determine the derivative of Eq. 38 and we get the following system:

$(\psi)^{0}: \frac{1}{2} \mathrm{q}_{0}^{2}+\mathrm{cq}_{0}=0$

$(\psi)^{1}: \alpha \mathrm{q}_{0} \mathrm{q}_{1}+\beta \mathrm{q}_{1}-\mathrm{cq}_{1}-\gamma \mathrm{q}_{1}=0$

$(\psi)^{2}:-\mathrm{cq}_{2}+\frac{1}{2} \alpha \mathrm{q}_{1}^{2}+4 \beta \mathrm{q}_{2}-3 \beta \mathrm{q}_{1}+15 \gamma \mathrm{q}_{1}-$

$16 \gamma q_{2}+\alpha q_{0} q_{1}=0$

$(\psi)^{3}: \alpha q_{0} q_{3}+\alpha q_{1} q_{2}+2 \beta q_{1}-10 \beta q_{2}+9 \beta q_{3}-$

$\mathrm{cq}_{3}-50 \gamma \mathrm{q}_{1}+130 \gamma \mathrm{q}_{2}-81 \gamma \mathrm{q}_{3}=0$

$(\psi)^{4}: \frac{1}{2} \alpha q_{2}^{2}+6 \beta q_{2}-\mathrm{cq}_{4}-21 \beta q_{3}+525 \gamma q_{3}-$

$256 \gamma q_{4}-330 \gamma q_{2}+60 \gamma q_{1}+\alpha q_{1} q_{3}+\alpha q_{0} q_{4}+$

$16 \beta \mathrm{q}_{4}=0$

$(\psi)^{5}: \alpha \mathrm{q}_{1} \mathrm{q}_{4}+\alpha \mathrm{q}_{2} \mathrm{q}_{3}+12 \beta \mathrm{q}_{3}-36 \beta \mathrm{q}_{4}-24 \gamma \mathrm{q}_{1}+$

$336 \gamma q_{2}-1164 \gamma q_{3}+1476 \gamma q_{4}=0$

$(\psi)^{6}: \frac{1}{2} \alpha q_{2}^{2}-120 \gamma q_{2}+1080 \gamma q_{3}-3020 \gamma q_{4}+$

$20 \beta \mathrm{q}_{4}+\alpha \mathrm{q}_{2} \mathrm{q}_{4}=0$

$(\psi)^{7}: \alpha q_{1} q_{4}-360 \gamma q_{3}+2640 \gamma q_{4}=0$

$(\psi)^{8}: \frac{1}{2} \alpha q_{4}^{2}-840 \gamma q_{4}=0$

Solving the system of equations from $(\psi)^{0}$ to $(\psi)^{8}$ by using the Maple, we get:

\section{Status 1:}

$\mathrm{q}_{0}=0, \mathrm{q}_{1}=0, \mathrm{q}_{2}=1680 \gamma / \alpha, \mathrm{q}_{3}=-3360 \gamma / \alpha, \mathrm{q}_{4}=1680 \gamma / \alpha$, $\beta=13 \gamma, c=36 \gamma$

$$
\begin{aligned}
& \mathrm{u}(\mathrm{x}, \mathrm{t})=\frac{105 \gamma}{\alpha}-\frac{210 \gamma}{\alpha} \tanh ^{2}\left(\frac{\mathrm{x}-36 \gamma \mathrm{t}}{2}\right)+ \\
& \frac{105 \gamma}{\alpha} \tanh ^{4}\left(\frac{\mathrm{x}-36 \gamma \mathrm{t}}{2}\right)
\end{aligned}
$$

\section{Status 2 A:}

$$
\begin{aligned}
& \beta=\frac{-13}{2} \gamma+\frac{39}{62} \mathrm{I} \gamma \sqrt{31}, \mathrm{q}_{0}=0, \mathrm{q}_{1}=\frac{840}{\alpha}\left(\frac{-1}{2} \gamma+\frac{1}{62} \gamma \mathrm{I} \sqrt{31}\right) \\
& \mathrm{q}_{2}=\frac{840}{\alpha}\left(\frac{-5}{2} \gamma+\frac{1}{62} \gamma \mathrm{I} \sqrt{31}\right), \mathrm{q}_{3}=\frac{-3360 \gamma}{\alpha}, \mathrm{q}_{4} \frac{1680 \gamma}{\alpha} \\
& C=\frac{-15}{2} \gamma+\frac{39}{62} \mathrm{I} \gamma \sqrt{31}
\end{aligned}
$$

$$
\begin{aligned}
& u(x, t)=\frac{105 \gamma}{\sqrt{31} \alpha} I \gamma \operatorname{sech}^{2}\left(\frac{x-\left(\frac{-15}{2} \gamma+\frac{39}{62} I \gamma \sqrt{31} t\right)}{2}\right)- \\
& \left.\frac{105 \gamma}{\alpha} \tanh ^{2}\left(\frac{\left.x-\left(\frac{-15}{2} \gamma+\frac{39}{62} I \gamma \sqrt{31}\right)\right)}{2}\right)+\frac{105 \gamma}{\alpha}\right) \\
& \tanh ^{4}\left(\frac{x-\left(\frac{-15}{2} \gamma+\frac{39}{62} I \gamma \sqrt{31}\right) t}{2}\right)
\end{aligned}
$$

\section{Status 2 B:}

$$
\begin{aligned}
& \beta=\frac{-13}{2} \gamma-\frac{39}{62} \mathrm{I} \gamma \sqrt{31}, \mathrm{q}_{0}=0, \mathrm{q}_{1}=\frac{840}{\alpha}\left(\frac{-1}{2} \gamma-\frac{1}{62} \gamma \mathrm{I} \sqrt{31}\right), \\
& \mathrm{q}_{2}=\frac{840}{\alpha}\left(\frac{-5}{2} \gamma-\frac{1}{62} \gamma \mathrm{I} \sqrt{31}\right), \mathrm{q}_{3}=\frac{-3360 \gamma}{\alpha}, \mathrm{q}_{4}=\frac{1680 \gamma}{\alpha}, \\
& \mathrm{C}=\frac{-15}{2} \gamma-\frac{39}{62} \mathrm{I} \gamma \sqrt{31} \\
& \mathrm{u}(\mathrm{x}, \mathrm{t})=-\frac{105 \gamma}{\sqrt{31} \alpha} \mathrm{I} \gamma \operatorname{sech}^{2}\left(\frac{\mathrm{x}-\left(\frac{-15}{2} \gamma-\frac{39}{62} \mathrm{I} \gamma \sqrt{31}\right)}{2}\right)- \\
& \frac{105 \gamma}{\alpha} \tanh ^{2}\left(\frac{\mathrm{x}-\left(\frac{-15}{2} \gamma-\frac{39}{62} \mathrm{I} \gamma \sqrt{31}\right)}{0} \mathrm{t}\right)+ \\
& \frac{105 \gamma}{\alpha} \tanh ^{4}\left(\frac{\mathrm{x}-\left(\frac{-15}{2} \gamma-\frac{39}{62} \mathrm{I} \gamma \sqrt{31}\right)}{2} \mathrm{t}\right)
\end{aligned}
$$

Status 3:

$$
\begin{aligned}
& \beta=13 \gamma, \mathrm{q}_{0}=\frac{-72 \gamma}{\alpha}, \mathrm{q}_{1}=0, \mathrm{q}_{2}=\frac{1680 \gamma}{\alpha} \\
& \mathrm{q}_{3}=\frac{-3360 \gamma}{\alpha}, \mathrm{q}_{4}=\frac{1680 \gamma}{\alpha}, \mathrm{C}=-36 \gamma \\
& \mathrm{u}(\mathrm{x}, \mathrm{t})=\frac{33 \gamma}{\alpha}-\frac{210 \gamma}{\alpha} \tanh ^{2}\left(\frac{\mathrm{x}+36 \gamma}{2} \mathrm{t}\right)+ \\
& \frac{105 \gamma}{\alpha} \tanh ^{4}\left(\frac{\mathrm{x}+36 \gamma}{2} \mathrm{t}\right)
\end{aligned}
$$


Status 4 A:

$$
\begin{aligned}
& \beta=\frac{-13}{2} \gamma+\frac{39}{62} \mathrm{I} \gamma \sqrt{31}, \mathrm{q}_{0}=\frac{6}{\alpha}\left(\frac{5}{2} \gamma+\frac{13}{62} \gamma \mathrm{I} \sqrt{31}\right), \\
& \mathrm{q}_{1}=\frac{-840}{\alpha}\left(\frac{1}{2} \gamma+\frac{1}{62} \gamma \mathrm{I} \sqrt{31}\right), \\
& \mathrm{q}_{2}=\frac{840}{\alpha}\left(\frac{5}{2} \gamma+\frac{1}{62} \gamma \mathrm{I} \sqrt{31}\right), \mathrm{q}_{3}=\frac{-3360 \gamma}{\alpha}, \\
& \mathrm{q}_{4}=\frac{1680 \gamma}{\alpha}, \mathrm{C}=\frac{15}{2} \gamma+\frac{39}{62} \mathrm{I} \gamma \sqrt{31} \\
& \mathrm{u}(\mathrm{x}, \mathrm{t})=\frac{105 \gamma}{\sqrt{31} \alpha} \mathrm{I} \gamma \tanh ^{2}\left(\frac{\mathrm{x}-\left(\frac{-15}{2} \gamma+\frac{39}{62} \mathrm{I} \gamma \sqrt{31}\right)}{2} \mathrm{t}\right)- \\
& \frac{105 \gamma}{\alpha} \tanh ^{2}\left(\frac{\mathrm{x}-\left(\frac{-15}{2} \gamma+\frac{39}{62} \mathrm{I} \gamma \sqrt{31}\right)}{2} \mathrm{t}\right)+ \\
& \frac{105 \gamma}{\alpha} \tanh ^{4}\left(\frac{\mathrm{x}-\left(\frac{-15}{2} \gamma+\frac{39}{62} \mathrm{I} \gamma \sqrt{31}\right)}{2} \mathrm{t}\right)+\frac{15 \gamma}{\alpha}- \\
& \frac{66 \mathrm{I} \gamma}{\sqrt{31} \alpha}=0
\end{aligned}
$$

\section{Status 4 B:}

$$
\begin{aligned}
& \beta=\frac{-13}{2} \gamma-\frac{39}{62} \mathrm{I} \gamma \sqrt{31}, \mathrm{q}_{0}=\frac{6}{\alpha}\left(\frac{5}{2} \gamma-\frac{13}{62} \gamma \mathrm{I} \sqrt{31}\right), \\
& \mathrm{q}_{1}=\frac{840}{\alpha}\left(\frac{1}{2} \gamma-\frac{1}{62} \gamma \mathrm{I} \sqrt{31}\right), \mathrm{q}_{2}=\frac{840}{\alpha}\left(\frac{-5}{2} \gamma-\frac{1}{62} \gamma \mathrm{I} \sqrt{31}\right), \\
& \mathrm{q}_{3}=\frac{-3360 \gamma}{\alpha}, \mathrm{q}_{4}=\frac{1680 \gamma}{\alpha}, \mathrm{C}=\frac{15}{2} \gamma-\frac{39}{62} \mathrm{I} \gamma \sqrt{31} \\
& \mathrm{u}(\mathrm{x}, \mathrm{t})=-\frac{105 \gamma}{\sqrt{31} \alpha} \mathrm{I} \gamma \tan \mathrm{h}^{2}\left(\frac{\mathrm{x}-\left(\frac{-15}{2} \gamma-\frac{39}{62} \mathrm{I} \gamma \sqrt{31}\right)}{2} \mathrm{t}\right)- \\
& \frac{105 \gamma}{\alpha} \tanh ^{2}\left(\frac{\left.\left.\mathrm{x}-\left(\frac{-15}{2} \gamma-\frac{39}{62} \mathrm{I} \gamma \sqrt{31}\right)\right) \mathrm{t}\right)+}{0}\right) \\
& \frac{105 \gamma}{\alpha} \tanh ^{4}\left(\frac{\mathrm{x}-\left(\frac{-15}{2} \gamma-\frac{39}{62} \mathrm{I} \gamma \sqrt{31}\right)}{2} \mathrm{t}\right)+\frac{15 \gamma}{\alpha}+\frac{66 \mathrm{I} \gamma}{\sqrt{31} \alpha}
\end{aligned}
$$

\section{RESULTS AND DISCUSSION}

Graphical representations of the solutions: Maple software is used to plot the obtain result. Figure 1-3 show the graphical representation of the solution (Table 3).

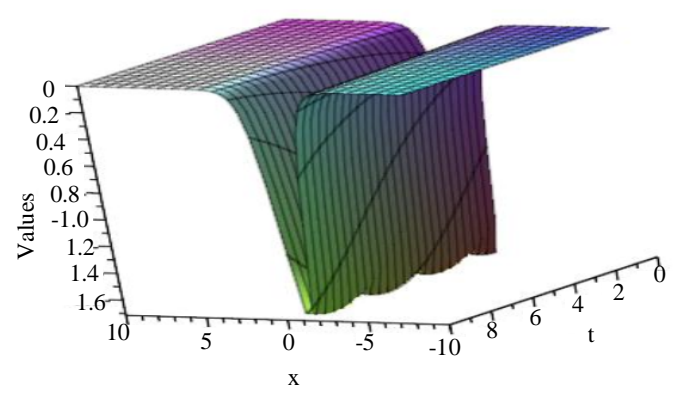

Fig. 1: The solitary wave 3D graphics of Eq. 13 for $a=2, b=2, k=2, x=-10, \ldots, 10$ and $t=0, \ldots, 10$
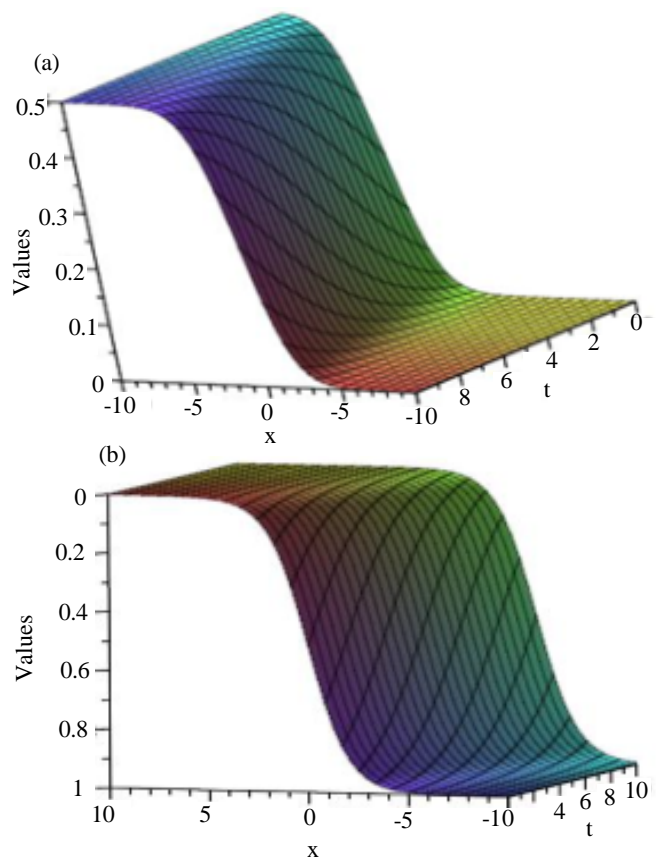

Fig. 2: a) The solitary wave 3D graphics of Eq. 26 for $\mathrm{x}=-10, \ldots, 10$ and $\mathrm{t}=0, \ldots, 10$ and $\mathrm{b}$ ) The solitary wave 3D graphics of Eq. 27 for $x=-10, \ldots, 10$ and $\mathrm{t}=0, \ldots, 10$

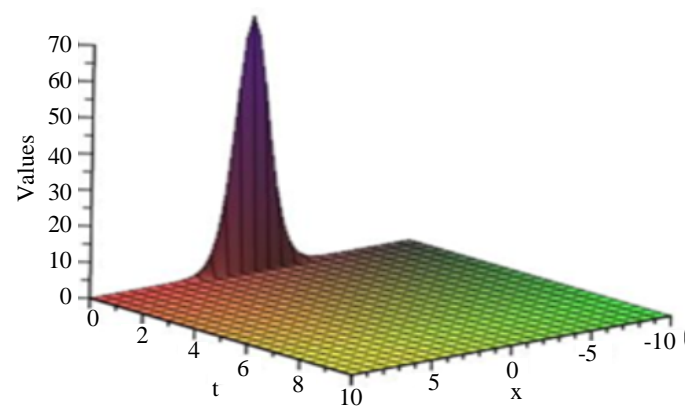

Fig. 3: The solitary wave 3D graphics of Eq. 39 for $\alpha=3$, $\gamma=3, x=-10, \ldots, 10$ and $t=0, \ldots, 10$ 
Table 3: Comparison of proposed solutions with solution of (G $\left(\mathrm{G}^{\prime} / \mathrm{G}\right)$-expansion method (Wazwaz, 2006)

\begin{tabular}{ll}
\hline Solution of $\left(\mathrm{G}^{\prime} / \mathrm{G}\right)$-expansion method & Proposed homogeneous balance solution \\
\hline If $\beta=4, \gamma=-1 / 13$ then Eq. 33 becomes & If $\gamma=16 / 13$ then, Eq. 42 \\
$\mathrm{u}(\mathrm{x}, \mathrm{t})=\frac{528}{13 \alpha}-\frac{3360}{13 \alpha} \tanh ^{2}\left(\mathrm{x}+\frac{576}{13} \mathrm{t}\right)+$ & $\mathrm{u}(\mathrm{x}, \mathrm{t})=\frac{528}{13 \alpha}-\frac{3360}{13 \alpha} \tanh ^{2}\left(\mathrm{x}+\frac{576}{13} \mathrm{t}\right)+$ \\
$\frac{1680}{13 \alpha} \tanh ^{4}\left(\mathrm{x}+\frac{576}{13} \mathrm{t}\right)$ & $\frac{1680}{13 \alpha} \tanh ^{4}\left(\mathrm{x}+\frac{576}{13} \mathrm{t}\right)$
\end{tabular}

\section{CONCLUSION}

In this research study, the homogeneous balance method is proposed to find the an exact solution for nonlinear equations such as the Generalized Regularized Long Wave (GRLW) equation, the MDWW equation and the kawahara equation. The proposed method has high efficiency and practicality in finding exact solutions. The obtained result was compared with other proposed methods in the literature under special condition. The finding reveals that our proposed method is less complex and more efficient than the methods used in the literature. Additionally, the proposed method is capable to be applied to wide range of different selected equation and the result would be useful in different applied situation.

\section{ACKNOWLEDGEMENT}

This research is supported by the University of Kirkuk.

\section{REFERENCES}

Abazari, R., 2010. Application of ( $\left.\mathrm{G}^{\prime} / \mathrm{G}\right)$-expansion method to travelling wave solutions of three nonlinear evolution equation. Comput. Fluids, 39: 1957-1963.

Alzaidy, J.F., 2013. The $\left(\mathrm{G}^{\prime} / \mathrm{G}\right)$-expansion method for finding traveling wave solutions of some nonlinear PDES in mathematical physics. Intl. J. Mod. Eng. Res., 3: 369-376.

Antonova, M. and A. Biswas, 2009. Adiabatic parameter dynamics of perturbed solitary waves. Commun. Nonlinear Sci. Numer. Simul., 14: 734-748.

Benjamin, R.T., J.L. Bona and J.J. Mahony, 1972. Model equations for long waves in nonlinear dispersive systems. Philos. Trans. R. Soc. London, 272: 47-78.

Biswas, A., 2010. Solitary waves for power-law regularized long-wave equation and $\mathrm{R}(\mathrm{m}, \mathrm{n})$ equation. Nonlinear Dyn., 59: 423-426.

Bridges, T.J. and G. Derks, 2002. Linear instability of solitary wave solutions of the Kawahara equation and its generalizations. SIAM. J. Math. Anal., 33: 1356-1378.
Elwakil, S.A., S.K. El-Labany, M.A. Zahran and R. Sabry, 2003. Exact travelling wave solutions for the generalized shallow water wave equation. Chaos Solitons Fractals, 17: 121-126.

Elwakil, S.A., S.K. El-Labany, M.A. Zahran and R. Sabry, 2004. New exact solutions for a generalized variable coefficients 2D KdV equation. Chaos Solitons Fractals, 19: 1083-1086.

Fan, E., 2000. Two new applications of the homogeneous balance method. Phys. Lett., 265: 353-357.

Fan, E., 2003. An algebraic method for finding a series of exact solutions to integrable and nonintegrable nonlinear evolution equations. J. Phys. Math. Gen., 36: 7009-7026.

Kawahara, T., 1972. Oscillatory solitary waves in dispersive media. J. Phys. Soc. Jpn., 33: 260-264.

Khalfallah, M., 2009. New exact traveling wave solutions of the $(3+1)$ dimensional Kadomtsev-Petviashvili (KP) equation. Commun. Nonlinear Sci. Numer. Simul., 14: 1169-1175.

Neyrame, A., A. Roozi, S.S. Hosseini and S.M. Shafiof, 2010. Exact travelling wave solutions for some nonlinear partial differential equations. J. King Saud Univ. Sci., 22: 275-278.

Ozis, T. and I. Aslan, 2010. Application of the G'/Gexpansion method to Kawahara type equations using symbolic computation. Appl. Math. Comput., 216: 2360-2365.

Peregrine, D.H., 1966. Calculations of the development of an undular bore. J. Fluid Mech., 25: 321-330.

Razborova, P., H. Triki and A. Biswas, 2013. Perturbation of dispersive shallow water waves. Ocean Eng., 63: 1-7.

Wang, M., X. Li and J. Zhang, 2008. The ( $\left.G^{\prime} G\right)$-expansion method and travelling wave solutions of nonlinear evolution equations in mathematical physics. Phys. Lett., 372: 417-423.

Wang, M., Y. Zhou and Z. Li, 1996. Application of a homogeneous balance method to exact solutions of nonlinear equations in mathematical physics. Phys. Lett., 216: 67-75.

Wang, M.L., 1995. Solitary wave solutions for variant Boussinesq equations. Phys. Lett. A., 199: 169-172. 
Wazwaz, A.M., 2006. New solitary wave solutions to the Kuramoto-Sivashinsky and the Kawahara equations. Appl. Math. Comput., 182: 1642-1650.

Zayed, E.M. and K.A. Alurrfi, 2014. The homogeneous balance method and its applications for finding the exact solutions for nonlinear evolution equations. Ital. J. Pure Appl. Math., 33: 307-318.
Zayed, E.M.E. and A.H. Arnous, 2012. DNA dynamics studied using the homogeneous balance method. Chin. Phys. Lett., 29: 10-12.

Zhao, X., L. Wang and W. Sun, 2006. The repeated homogeneous balance method and its applications to nonlinear partial differential equations. Chaos Solitons Fractals, 28: 448-453. 